\title{
Perfil epidemiológico de patologías apicales en pacientes que acuden a centros de atención odontológica en Cuenca (Ecuador).
}

\section{Epidemiological profile of apical pathologies in patients attending dentistry care centers in Cuenca (Ecuador).}

\author{
Cedillo-Orellana Ivanna* ${ }^{1}$,Torracchi-Carrasco Esteban ${ }^{2}$ Ochoa-Vázquez Boris, ${ }^{3}$ Cedillo-Orellana Antonella. ${ }^{4}$ \\ ${ }^{1}$ Docente de la Universidad Católica de Cuenca, Especialista en Endodoncia. Cuenca, Ecuador. \\ ${ }^{2}$ Docente de la carrera de Odontología de la Universidad Católica de Cuenca. Ecuador. \\ ${ }^{3}$ Especialista en Rehabilitación Oral Avanzada e Implantología. Ecuador. \\ ${ }^{4}$ Residente del Posgrado de Periodoncia de la Universidad Latinoamericana de México. \\ *scedilloo@ucacue.edu.ec
}

\begin{abstract}
Resumen
Objetivo: Definir el perfil epidemiológico de las patologías apicales en la ciudad de Cuenca-Ecuador en el período marzo 2017 - febrero 2018. Materiales y Métodos: Se realizó un estudio retrospectivo, basado en el análisis de 785 fichas clínicas del área de endodoncia correspondientes a pacientes atendidos en centros de atención odontológica en la ciudad de Cuenca (Ecuador) en el período marzo 2017-febrero 2018. Resultados: : El 29,17\% de los pacientes atendidos presentaron algún tipo de patología apical. Estas patologías se presentan principalmente en pacientes de sexo femenino, con edades comprendidas entre los 26 y los 35 años y cuya ocupación se clasifica dentro de la categoría "ocupaciones elementales". Conclusión: Existe una moderada frecuencia de patologías apicales en los pacientes que son remitidos al área de endodoncia de centros de atención odontológica de la ciudad de Cuenca. Esto muy probablemente es el resultado de patologías pulpares no atendidas oportunamente.
\end{abstract}

Palabras clave: Perfil epidemiológico, Endodoncia, Periodontitis, Absceso apical, Osteítis condensante.

\begin{abstract}
Aim: To define the epidemiological profile of apical pathologies in the city of Cuenca-Ecuador, during the period March 2017 - February 2018. Materials and Methods: A retrospective study was conducted, based on the analysis of 785 clinical records of the endodontics area corresponding to patients treated in dental care centers in the city of Cuenca (Ecuador) in the period March 2017-February 2018. Results: Approximately one third (29.17\%) of the patients presented some type of apical pathology. These pathologies occur mainly in female patients, aged between 26 and 35 years and whose occupation is classified within the category "elementary occupations". Conclusion:There is a moderate frequency of apical pathologies in patients who are referred to the endodontics area of dental care centers in the city of Cuenca. This is most likely the result of pulpal pathologies not treated in a timely manner.
\end{abstract}

Key words: Epidemiological profile, Endodontics, Periodontitis, Apical abscess, Condensing osteítis.

\section{INTRODUCCIÓN}

La patología apical (PA) es un trastorno inflamatorio crónico de los tejidos perirradiculares causado por una infección de origen endodóntico generalmente ocasionada por microorganismos patógenos de la caries dental que se diseminan a lo largo de los conductos radiculares alcanzando los tejidos apicales. Es la consecuencia de un encuentro dinámico entre los microorganismos que infectan el conducto radicular y los componentes del sistema inmunitario del hospedador (por ej., células inmunoinflamatorias y mediadores solubles) que produce una inflamación local, reabsorción de tejidos duros y destrucción de otros tejidos periapicales. ${ }^{1,2}$ La prevalencia de las PA aumenta con la edad de los pacientes y varía de $33 \%$ a $62 \%{ }^{3,8}$ En las PA, la capacidad del hospedador para regenerar y reparar el tejido dañado está comprometida por la presencia de contaminación bacteriana, pudiendo convertirse en un proceso crónico; en ocasiones, cuando la respuesta inmunitaria no es eficaz, se pueden originar complicaciones sistémicas severas a menos que se inicie un tratamiento clínico oportuno y eficaz para eliminar los agentes infeciosos. ${ }^{9,10}$

Las PA junto con las patologías pulpares son las 
principales enfermedades bucodentales por las que los pacientes acuden a consulta de urgencia, seguido de los traumatismos dentales, problemas periodontales y causas iatrogénicas e idiopáticas. ${ }^{11,12}$

Al ser la patología apical de naturaleza progresiva, los hallazgos clínicos y radiográficos varían de acuerdo a la etapa de la enfermedad que se encuentre cursando el paciente al momento del examen. ${ }^{13}$ Por lo que con fines de establecer un diagnóstico adecuado, la Asociación Americana de Endodoncia (AAE) desarrolló una un consenso de terminología diagnóstica basada en los síntomas del paciente y los resultados de pruebas clínicas que tiene mayor aplicabilidad práctica clasificando las patologías apicales en: tejidos apicales normales, periodontitis apical sintomática, periodontitis apical asintomática, absceso apical crónico, absceso apical agudo y osteítis condensante. ${ }^{14,15}$

Con base a lo anterior se planteó este estudio con el fin de determinar la frecuencia de las patologías periapicales en pacientes atendidos en centros de atención odontológica de la ciudad de Cuenca y al mismo tiempo identificar factores de riesgo asociados, tales como el sexo, la edad y ocupación laboral, debido a que no existen registros de trabajos de investigación publicados en Ecuador acerca de este tema.

\section{MATERIALES Y MÉTODOS}

El presente estudio corresponde a un trabajo retrospectivo de enfoque cuantitativo con diseño descriptivo, basado en la revisión de datos clínicos como diagnóstico, sexo, edad y ocupación de 785 pacientes atendidos en el área de endodoncia en la ciudad de Cuenca en el período marzo 2017-febrero 2018, cuyas fichas están archivadas en dicho centro odontológico.

El diagnóstico fue establecido en base a la terminología del "Consensus Conference Recommended Diagnostic Terminology", de la AAE del 2009 el cual clasifica las PA de la siguiente manera: tejidos apicales normales, periodontitis apical sintomática, periodontitis apical asintomática, absceso apical agudo, absceso apical crónico, osteítis condensante. ${ }^{15}$

En el estudio se incluyeron pacientes de ambos sexos, con edades comprendidas entre 15 y 70 años. Para el criterio ocupación se empleó la "Clasificación Nacional de Ocupaciones, CIUO 08" del Instituto Nacional de Estadística y Censos del Ecuador (INEC), que establece las siguientes categorías: 1 directores y gerentes, 2 profesionales científicos e intelectuales, 3 técnicos y profesionales de nivel medio, 4 personal de apoyo administrativo, 5 trabajadores de los servicios y vendedores de comercios y mercados, 6 agricultores y trabajadores calificados agropecuarios, forestales y pesqueros, 7 oficiales, operarios y artesanos de artes mecánicas y de otros oficios, 8 operadores de instalaciones, máquinas y ensambladores, 9 ocupaciones elementales y 0 ocupaciones militares. ${ }^{17}$

Para el ingreso de los datos se utilizó el programa de libre acceso EPI INFO (v.7.2) y posteriormente, para determinar posibles diferencias entre los grupos estudiados, se aplicó el test de Chi-cuadrado con el cálculo del valor p basado en el modelo de Monte Carlo (con 10000 simulaciones), o el test de Chi-cuadrado de Pearson. ${ }^{18}$

\section{RESULTADOS}

Los hallazgos revelaron que el 29,17\% de los pacientes atendidos presentaron patologías apicales (Tabla 1). De estas, la más frecuente resultó ser la periodontitis apical asintomática, mientras que la menos frecuente fue la osteítis condensante por lo que, si bien esta enfermedad es una patología apical, no se presentó en ninguna de las personas sujetas a esta investigación por lo que no fue considerada dentro del análisis. Los pacientes de sexo femenino fueron más afectados por las patologías pulpares $(52.83 \%)$ que los de sexo masculino $(47.16 \%)$ (Chi cuadrado $=6,627 ; \mathrm{p}=0,08480)$ (Tabla 2). Asimismo, el rango de edad más afectado por estas patologías fue el correspondiente al grupo de 26 a 35 años $(29,69 \%)($ Chi cuadrado $=20,55 ; \mathrm{p}=0,1477)($ Tabla 3). Finalmente, los pacientes con "ocupaciones elementales" (como vender mercancías en las calles, brindar servicios de portería y vigilancia de inmuebles y bienes, limpiar, lavar, planchar ropa y ejecutar tareas simples relacionadas con la minería, la agricultura o la pesca, la construcción o las obras públicas y las industrias manufactureras) fueron los que presentaron patologías pulpares con mayor frecuencia $(46,72 \%)($ Chi cuadrado $=23,55 ; \mathrm{p}=0,1653)($ Tabla 4$)$.

\begin{tabular}{lrr}
\hline \multicolumn{1}{c}{ Diagnóstico } & \multicolumn{1}{c}{$\mathbf{N}^{\circ}$} & \multicolumn{1}{c}{$\%$} \\
\hline PATOLOGÍA APICAL & & \\
Periodontitis Apical Sintomática & 47 & 20,52 \\
Periodontitis Apical Asintomática & 81 & 35,37 \\
Absceso Apical Agudo & 32 & 13,97 \\
Absceso Apical Crónico & 69 & 30,13 \\
TOTAL & $\mathbf{2 2 9}$ & $\mathbf{1 0 0}$ \\
\hline
\end{tabular}

Fig. 1. Frecuencia de patologías apicales en pacientes atendidos en Cuenca en el período marzo 2017 - febrero 2018

\begin{tabular}{lrrrr}
\hline \multicolumn{1}{c}{ Diagnóstico } & Hombres & $\%$ & Mujeres & \% \\
\hline PATOLOGíA APICAL & & & & \\
Periodontitis Apical Sintomática & 30 & 27,78 & 17 & 14,05 \\
Periodontitis Apical Asintomática & 35 & 32,41 & 46 & 38,02 \\
Absceso Apical Agudo & 14 & 12,96 & 18 & 14,88 \\
Absceso Apical Crónico & 29 & 26,85 & 40 & 33,06 \\
TOTAL & $\mathbf{1 0 8}$ & $\mathbf{1 0 0 , 0 0}$ & $\mathbf{1 2 1}$ & $\mathbf{1 0 0 , 0 0}$ \\
\hline
\end{tabular}

Fig. 2. Patologías apicales en función del sexo. 


\begin{tabular}{lllllllll}
\hline \multicolumn{1}{c}{ Diagnóstico } & \multicolumn{8}{c}{ Rangos de Edad } \\
& $\mathbf{1 5 - 2 5}$ & $\mathbf{2 6 - 3 5}$ & $\mathbf{3 6 - 4 5}$ & $\mathbf{4 6 - 5 5}$ & $\mathbf{5 6 - 6 5}$ & $\mathbf{> 6 5}$ & $\begin{array}{l}\text { Total } \\
\text { general }\end{array}$ \\
\hline $\begin{array}{l}\text { Periodontitis apical } \\
\text { sintomática }\end{array}$ & 15 & 13 & 10 & 6 & 3 & 0 & 47 \\
$\begin{array}{l}\text { Periodontitis apical } \\
\text { asintomática }\end{array}$ & 14 & 31 & 15 & 15 & 3 & 3 & 81 \\
Absceso apical agudo & 16 & 5 & 3 & 5 & 2 & 1 & 32 \\
Absceso apical crónico & 15 & 19 & 17 & 13 & 3 & 2 & 69 \\
Total general & $\mathbf{6 0}$ & $\mathbf{6 8}$ & $\mathbf{4 5}$ & $\mathbf{3 9}$ & $\mathbf{1 1}$ & $\mathbf{6}$ & $\mathbf{2 2 9}$ \\
\hline
\end{tabular}

Fig. 3. Patologías apicales por rango de edad.

\begin{tabular}{lccccccccc}
\hline Diagnóstico & $\mathbf{3}$ & $\mathbf{4}$ & $\mathbf{5}$ & $\mathbf{6}$ & $\mathbf{7}$ & $\mathbf{8}$ & $\mathbf{9}$ & NA & Total general \\
\hline $\begin{array}{l}\text { Periodontitis apical } \\
\text { sintomática }\end{array}$ & 1 & 2 & 6 & 0 & 7 & 3 & 28 & 0 & 47 \\
$\begin{array}{l}\text { Periodontitis apical } \\
\text { asintomática }\end{array}$ & $\mathbf{8}$ & 3 & 19 & 1 & 10 & 7 & 30 & 3 & 81 \\
Absceso apical agudo & 2 & 0 & 4 & 2 & 4 & 2 & 18 & 0 & 32 \\
Absceso apical & 6 & 3 & 5 & 1 & 11 & 11 & 31 & 1 & 69 \\
crónico & $\mathbf{1 7}$ & $\mathbf{8}$ & $\mathbf{3 4}$ & $\mathbf{4}$ & $\mathbf{3 2}$ & $\mathbf{2 3}$ & $\mathbf{1 0 7}$ & $\mathbf{4}$ & $\mathbf{2 2 9}$ \\
\hline Total general & & & & & & & & & \\
\hline
\end{tabular}

Fig. 4. Patologías apicales según la ocupación de los pacientes.

\section{DISCUSIÓN}

Con la intención de definir la frecuencia de las patologías apicales en la ciudad de Cuenca-Ecuador en el período marzo 2017 - febrero 2018, se llevó a cabo el presente estudio. El mismo reveló que aproximadamente un tercio $(29,17 \%)$ de los pacientes remitidos al área de endodoncia padecen este tipo de patologías. Se trata de la continuación de un trabajo anterior relacionado con el perfil epidemiológico de las patologías pulpares. ${ }^{16}$

El antecedente más cercano al presente trabajo es una tesis de grado realizada por Galán y col. ${ }^{19}$ la cuál reveló un $36 \%$ de patologías apicales, con mayor predominancia en pacientes de sexo femenino y en edades comprendidas entre 20 a 64 años; datos similares a los obtenidos en el presente estudio. No obstante, dicho trabajo no ha sido publicado.

En relación a la posible asociación existente entre las patologías apicales y el sexo de los pacientes, los más afectados en este estudio fueron las mujeres (52,83\%), aunque la diferencia no es estadísticamente significativa. Estos resultados coinciden con estudios realizados en Ecuador, ${ }^{19}$ Venezuela, ${ }^{20}$ Cuba, ${ }^{?}$ Chile ${ }^{24}$ y México ${ }^{25}$

En algunos de estos estudios se señala que, en dichos países, las mujeres tuvieron mayor oportunidad de acudir a consulta dental que los varones ya que al desempeñar en su mayoría labores domésticos disponían de flexibilidad de horario. $^{20,21}$ Otro estudio indica que esta situación podría atribuirse a que las mujeres se preocupan más por su salud bucal, demostrando mayor interés por recibir un tratamiento odontológico para mejorar su estética y funcionalidad. ${ }^{24}$ De manera que aún es necesario profundizar más en este aspecto con el fin de poder establecer, de manera fehaciente, una posible relación entre el sexo de los pacientes y la manifestación de este tipo de patologías.

Los datos obtenidos en relación con la edad reflejan que las patologías apicales se presentaron con mayor frecuencia en edades comprendidas entre los 26 y los 35 años. Sin embargo, estos resultados son diferentes a los reportados en otros estudios realizados en Cuba, ${ }^{23,26}$ Chile $^{24}$ y México; ${ }^{25,27}$ muy probablemente esto se deba a que los grupos etarios establecidos son diferentes entre estudios y la muestra no es homogénea en todos los grupos etáreos. Llama la atención en este estudio el hecho de que la mayor cantidad de pacientes que presentan patologías apicales corresponden al grupo de personas con "ocupaciones elementales". En general, estas personas desempeñan trabajos que consisten en vender mercancías en las calles, brindar servicios de portería y vigilancia de inmuebles y bienes, limpiar, lavar, planchar ropa y ejecutar tareas simples relacionadas con la minería, la agricultura o la pesca, la construcción o las obras públicas y las industrias manufactureras. ${ }^{28}$ Siendo que no existen estudios previos que analicen la posible relación entre las patologías pulpares y la ocupación de los pacientes, se podría argumentar que existe una relación entre la situación socioeconómica (ingresos, ocupación y nivel de educación) y la prevalencia y gravedad de las enfermedades bucodentales. En efecto, la falta de acceso a la educación determina menores posibilidades en el campo laboral y esto conlleva a menores ingresos económicos. Por lo tanto, por ser el acceso a servicios de salud dental de especialidad —necesarios en muchos de los casos - más costoso, estas personas deben acudir a servicios de salud pública que en la mayoría de casos ofrece tratamientos básicos. Esto podría por lo tanto acarrear complicaciones debidas a la aplicación de terapias no óptimas, tal y como lo plantean Marmot y cols. $^{29}$

Por lo que, este estudio fue realizado con el objetivo de aportar datos de interés acerca de las patologías apicales en el medio y así contribuir tanto en el desarrollo de programas de salud bucal como en el proceso de formación científica y académica.

\section{CONCLUSIÓN}

A través de este estudio hemos puesto en evidencia que las patologías apicales aquejan a un considerable porcentaje de la población cuencana que acude al área de endodoncia de centros odontológicos $(29,17 \%)$, revelando la deficiente e inoportuna atención de la patología pulpar. Además, se ha establecido que, en la mayoría de los casos, los pacientes que presentan estas patologías son mujeres en situación de ocupación elemental, cuya edad varía entre 26 y 35 años. Ya que hasta el momento poco se conocía sobre la epidemiología 
de estas patologías en la región de Cuenca, los resultados aportan datos importantes para futuras investigaciones, al mismo tiempo que confirman la imperiosa necesidad de actuar a nivel de cada uno de los factores predisponentes para reducir este importante problema de salud oral. Si tomamos en cuenta el hecho de que la principal causa de las patologías apicales es la patología pulpar no tratada, misma que presenta una alta prevalencia en nuestra población, se la puede considerar como un importante problema de salud bucodental, cuya oportuna intervención disminuiría la tasa de patologías apicales en pacientes del área de endodoncia.

\section{Referencias Bibliográficas}

1 Nair PNR. Apical periodontitis: a dynamic encounter between root canal infection and host response. Periodontol 2000 1997; 13:121-48.

2 Marton IJ, Kiss C. Overlapping protective and destructive regulatory pathways in apical periodontitis. J Endod 2014;40:155-63.

3 Hargreaves KM, Berman LH. Cohen's Pathways of the Pulp, 11th ed. St Louis: Elsevier Saunders; 2015.

4 Guías prácticas clínicas de enfermedades pulpares y periapicales. [serie en internet]. 2009 [citado 7 jul 2011]. Disponible en: http://endodonciaycirugiaapical.blogspot.com/2009/07/guiaspracticas-clinicasde.html

5 Calafell R, Ugalde A. Infecciones de los dientes y del hueso. Universidad Veracruzana Facultad de Odontología. Patología Bucal, 2011. Disponible en: http://www.slideshare.net/alufar/infecciones-de-losdientes- y-del-hueso

6 Gómez Y, García M. Comportamiento de las patologías pulpares y periapicales en los pacientes mayores de 19 años. Área Sur de Sancti Spíritus. Filial de Ciencias Médicas "Manuel P. Fajardo". Gaceta Médica Espirituana. 2009 [citado15 Ene 2010];11(1). Disponible en: http://bvs.sld.cu/revistas/gme/pub/vol.11.(1)_2/p2.htm

7 John I, Simon J, Walton R, Pashley D, Bakland L, Heithersay G, et al. Patología Pulpar: etiología y prevención. En: Ingle J, Bakland L. Endodoncia. México: Mc Graw-Hill; 2002. p. 95-175.

8 Alfonsin A. Caries y Enfermedad Periodontal. En: Rubinstein A, Terrasa S. Medicina Familiar y Práctica Ambulatoria. Argentina: Panamericana; 2006. p. 1300-06.

9 Walton RE, Johnson, WT. En Walton, RE y Torabinejad M. Principles and Practice of Endodontics. Philadelphia: Ed. Saunders; 2002. p. 240-67.

10 Torabinejad M, Walton R. Lesiones perirradiculares. En: Ingle J, Bakland L. Endodoncia. México: Mc Graw-Hill; 2002. p. 177-203.

11 Quiñones-Márquez D. Patologías pulpares y periapicales más frecuentes en urgencias en 2 clínicas estomatológicas. Rev Cubana Estomatol [Internet]. 2000 Ago 37( 2 ): 84-88.
Gómez-Porcegué Y, García-Sánchez ME. Comportamiento de las patologías pulpares y periapicales en los pacientes mayores de 19 años. GME. 2009; 11(1).

12 Abbott P. Classification, diagnosis and clinical manifestations of apical periodontitis. Endod Topic. 2004; 8(1): 3654.

13 Furzan S, Jiménez L. Prevalence of periapical pathologies in patients treated in the endodontic postgrade University of Carabobo. period 2010 - 2013. ORAL 2016; 17(55): 13911397

14 Orstavick D, Ford T. Essential Endodontics. London: Blackwell Science Ltd; 1999

15 AAAE Consensus Conference Recommended Diagnostic Terminology. J Endod.2009;35(12):1634.

16 Cedillo I, Torracchi E, Ochoa B, Cedillo E. Perfil epidemiológico de las patologías pulpares en pacientes que acuden a centros de atención odontológica en la ciudad de Cuenca (Ecuador). Revista O activa.2019; 4(3):15-20

17 Instituto Nacional de Estadísticas y Censos (INEC). Clasificación Nacional de Ocupaciones. (CIOU08). 2012.

18 Agresti, A. An Introduction to Categorical Data Analysis. 2 a ed. New York.2007:38.

19 Galán B, Pesántez J. Prevalencia de patología pulpar y periapical en pacientes atendidos en la Clínica de la Especialidad en Endodoncia de la Facultad de Odontología en el periodo 2011-2017 [Tesis de grado]. Cuenca: Universidad de Cuenca; 2019. 52p

20 Furzan Susana, Jiménez Liliana. Prevalencia de patologías periapicales en pacientes atendidos en el postgrado de endodoncia. Universidad de Carabobo. Período 2010-2013.Oral; 2016; 17(55): 1391-1397.

21 Fernández González María del Carmen, Valcárcel Llerandi Julio, Betancourt Núñez Marisette. Enfermedades pulpares y periapicales en trabajadores del Instituto Cubano de Oftalmología Ramón Pando Ferrer. Ciencias Clinicas y Patologicas; 2007; 1:7.

22 Grethe Bertrán HerreroI, José Luis Rosales Alonso.Lesiones pulpares y periapicales en la consulta de Urgencia Estomatológica. Clínica "Felipe Soto". 2010-2011.Revista Habanera de Ciencias Médicas 2013;13(1):94-100.

23 Mendiburu Zavala Celia Elena del Perpetuo Socorro, Peñaloza Cuevas Ricardo, Chuc Baas Inés del Rosario, Medina Peralta Salvador. Enfermedades pulpares y periapicales en estructuras dentales permanentes en pacientes con edades de seis-catorce años. Revista Cubana de Estomatología 2017;54(3).

24 León P, Ilabaca MJ, Alcota M, González FE. Frecuencia de periodontitis apical en tratamientos endodónticos de pregrado.Rev. Clin. Periodoncia Implantol. Rehabil. Oral; 2011; Vol. 4(3); 126-129

25 Mendiburu Zavala Celia Elena del Perpetuo Socorro, Medina Peralta Salvador, Chi Castillo Suemy Dolores. Enfermedades pulpares y periapicales en pacientes sistémicamente 
comprometidos en el Centro de Salud de Uayma, Yucatán, México. Revista Cubana de Estomatología 2016;53(4)

26 Fernández Collazo María Elena, Vila Morales Dadonim, Rodríguez Soto Agustín, Mesa González Dania Lucrecia, Pérez Clemente Norma. Lesiones periapicales agudas en pacientes adultos. Revista Cubana de Estomatología. 2012;26(2):107-116.

27 Mendiburu Zavala Celia Elena del Perpetuo Socorro, Medina Peralta Salvador, Peraza Dorantes Hernán Humberto. Prevalencia de enfermedades pulpares y periapicales en pacientes geriátricos: Mérida, Yucatán, México. Rev Cubana Estomatol. 2015;52(3).

28 Instituto Nacional de Estadísticas y Censos (INEC). Clasificación Nacional de Ocupaciones. (CIOU08). 2012.

29 World Health Organization. Equity, social determinants and public health programmes. Published 2010. Accessed 15 February.2018.

Recibido: 01 de Febrero del 2020

Aceptado: 15 de abril del 2020 
